\title{
ESTIMATION OF MICROALBUMIN IN TYPE - 2 DIABETES MELLITUS
}

Bharathi $\mathrm{R}^{1}$, Radhakishan $\mathrm{N}^{2}$, Naveen Kumar T33, Neetha $\mathrm{K}^{4}$, Srilatha $\mathrm{B}^{5}$

\section{HOW TO CITE THIS ARTICLE:}

Bharathi R, Radhakishan N, Naveen Kumar T, Neetha K, Srilatha B. "Estimation of Microalbumin in Type - 2 Diabetes Mellitus". Journal of Evolution of Medical and Dental Sciences 2014; Vol. 3, Issue 03, January 20; Page: 627-632, DOI: $10.14260 / \mathrm{jemds} / 2014 / 1877$

ABSTRACT: BACK GROUND: The objective of this study is to estimate microalbumin in type - 2 diabetes mellitus patients. METHODS: The sensitive marker for the detection of urine albumin excretion was done by estimation of morning urine sample by semi quantitative procedure by Bayerclinitek50 urine chemistry analyzer. For creatinine jaffes method, fasting blood sugar and post lunch blood sugar were estimated by glucometer and glycosylated hemoglobin $\left(\mathrm{HbA}_{1 \mathrm{c}}\right)$ was analyzed by Merck microlab 120 semi -automated analyzer. RESULTS: In the study significant increase in micro albumin was observed $193.50 \pm 73.66(\mathrm{P}<0.0001)$ in all the patients of type 2 diabetes mellitus which is the predictor of late development diabetic nephropathy. Para meters like fasting blood sugar, post lunch blood sugar, creatinine and glycosylated hemoglobin $\left(\mathrm{HbA}_{1 \mathrm{c}}\right)$ levels were increased above the normal range. CONCLUSION: In type 2 diabetes, microalbumin is the hallmark of subsequent diabetic nephropathy and a surrogate marker of cardiovascular diseases and increased cardiovascular mortality. In diabetic nephropathy patients, glomerular filtration rate is increased in early stage of the disease and glomerulopathy is an important renal structural change. Improving glycemic control in patients with type 2 diabetes may subsequently reduce the risk of complications.

KEY WORDS: Microalbumin, Diabetic nephropathy, Creatinine.

INTRODUCTION: In the year 2000, there were around 171 million people with diabetes globally, and by 2030, it is estimated that this number would increase to 366 million. ${ }^{1}$ As the number of persons with diabetes increases; the development of microvascular complications like retinopathy, nephropathy and neuropathy also rises. These microvascular complications are linked to the duration of diabetes mellitus, poor glycemic control and systolic hypertension. ${ }^{2}$ The magnitude of damage caused by these microvascular complications of diabetes stresses the need for sensitive markers of screening for retinopathy and nephropathy. The sensitive marker for the detection of diabetic nephropathy is to estimate excretion of microalbumin in urine; and for the detection of diabetic retinopathy (DR), to have a fundus evaluation after pupillary dilatation. 3,4

According to a report by the World Health Organization (WHO), the prevalence rates of nephropathy after 15 years of diabetes ranged between 17.7 and $56.6 \%$ in men and between 11.9 and $71 \%$ in women. Increased urinary protein excretion may be an early clinical manifestation of diabetic nephropathy. ${ }^{5-9}$

However, when assessing protein excretion, the urine dipstick is a relatively insensitive marker for initial increases in protein excretion, not becoming positive until protein excretion exceeds 300 to $500 \mathrm{mg} /$ day (upper limit of normal less than $150 \mathrm{mg} /$ day, with most individuals excreting less than $100 \mathrm{mg} /$ day).

Using a specific assay for albumin is a more sensitive technique. The normal rate of albumin excretion is less than $30 \mathrm{mg} /$ day $(20 \mathrm{mcg} / \mathrm{min})$; persistent albumin excretion between 30 and $300 \mathrm{mg} /$ day (20 to $200 \mathrm{mcg} / \mathrm{min}$ ) is called moderately increased albuminuria, may be indicative of 
early diabetic nephropathy, unless there is some coexistent renal disease. Albumin excretion above $300 \mathrm{mg} /$ day $(200 \mathrm{mcg} / \mathrm{min}$ ) is considered to represent severely increased albuminuria.

Although these cut-offs defining moderately increased albuminuria and severely increased albuminuria facilitate determining the risk for progression of nephropathy, the risk of developing overt diabetic nephropathy is probably directly related to albumin excretion rates at all levels.

The clinical significance, screening, and management of moderately increased albuminuria in patients with type 2 diabetes will be reviewed here. In addition to being a possible marker of early diabetic nephropathy, moderately increased albuminuria is also associated with cardiovascular disease in both diabetic and nondiabetic patients.

Diabetic Nephropathy progresses gradually and accounts for about $40 \%$ of ESRD (End stage Renal Disease).

The earliest clinical evidence of progressive diabetic nephropathy is repeated appearance of low but above normal levels of albumin in urine. Such a condition is referred as microalbuminuria.

Proteinuria is a key feature of diabetic nephropathy and strong predictor of speed progression towards end stage renal failure. Proteinuria independently associates with coronary heart disease in patients with diabetes and reflects not only renal impairment and a key pathogenic element of disease progression but also more generalized vascular damage.

Several genes have been proposed as possible candidates for the susceptibility to diabetic kidney disease. Polymorphism with in the angiotensin converting enzyme gene, the angiotensin types I receptor gene are associated with the development of diabetic nephropathy. 10

Diabetic nephropathy progresses gradually in early stages and there are no clinical signs and symptoms of glomerular changes. The onset of diabetic nephropathy can be diagnosed only by screening the suspected patients for microalbuminuria.

If diabetic nephropathy is diagnosed at the microalbuminuria stage, the further progress of nephropathy can be arrested with tight glycemic control, well controlled blood pressure and aggressive reno-protective therapy. This in turn can help to avoid ESRD.

METHODS: A population based case control study was done over a period of six months in Peoples Education Society Institute of Medical Sciences And Research, Kuppam, Andhra Pradesh after taking permission from Institutional Review Board and written informed consent was obtained from subjects as per Helsinki declaration. 30 diabetic patients and 30 control group either sex (M \&F) of age group 30-60 years with diabetes 10 years duration attending medicine outpatient department were included in the study. Microalbuminuria and parameters like fasting blood sugar, post prandial blood sugar, creatinine and glycosylated hemoglobin (HbA1c) were inclusion criteria. Individuals residing for a period of less than six months at the same residence, temporary residents (whose permanent residence is elsewhere), and those who cannot be contacted after five attempts by the social worker at their residence were excluded from the study.

Venous blood samples were collected after 12 hours of fasting for estimation of fasting blood sugar and for Postprandial blood sugar; samples were collected 2 hours after ingestion of food. The glucometer was calibrated every day and its reproducibility was assessed by measuring the blood glucose for the same patient six times and also with two machines. The collected data was scrutinized manually before its entry into the computer. 
Albuminuria estimation was done by a semi-quantitative procedure (Bayer Clinitek 50 Urine Chemistry Analyzer) with the first morning urine sample. ${ }^{11}$ The Clinitek Microalbumin strip contains two reagent areas that test for albumin and creatinine in urine and provide semi quantitative results. In this study, only the albumin results were used. The albumin test is based on dye binding, using a high-affinity sulfonaphthalein dye. At a constant $\mathrm{pH}$, the development of any blue color is due to the presence of albumin. The resulting strip color ranges from pale green to aqua blue. The results were read with the use of the Clinitek 50 Urine Chemistry Analyzer (Bayer Health- Care, Elkhart, Ind., USA). Sensitivity and specificity for microalbuminuria diagnosis have been reported to be 100 and $81 \%$ for Clinitek Microalbumin. ${ }^{12}$ The diagnostic performance of measuring UAC in a spot morning urine sample in predicting microalbuminuria in subsequent 24-hour urine collections has been reported to be satisfactory, and moreover comparable to that of measuring ACR. ${ }^{13}$

Subjects were considered to have microalbuminuria, if the Urinary Albumin Excretion (UAE) was between 30 and $300 \mathrm{mg} / 24$ hours. For estimation of creatinine Jaffe's kinetic method was followed. ${ }^{14}$

Glycosylated hemoglobin (HbA1c) fractions were estimated by using Merck Micro Lab 120 semi-automated analyzer (Bio-Rad DiaSTAT HbA1c Reagent Kit). ${ }^{15}$

Statistical analysis: All data analysis was completed using SPSS software. The results were expressed as Mean \pm SD. Two way analysis of variance (ANOVA) was used. P values less than 0.05 was considered statistically significant.

\section{RESULTS:}

\begin{tabular}{|c|c|c|}
\hline Sl. No & Patients and Control & Normal values (ADA) \\
\hline 1 & Microalbumin & $3-30 \mathrm{mg} / \mathrm{dl}$ \\
\hline 2 & FBS & $70-100 \mathrm{mg} / \mathrm{dl}$ \\
\hline 3 & PPBS & $180 \mathrm{mg} / \mathrm{dl}$ \\
\hline 4 & Creatinine & $0.6-1.5 \mathrm{mg} / \mathrm{dl}($ serum) \\
\hline 5 & HbA1C & Less $7.0 \%$ \\
\hline
\end{tabular}

\section{Table -1: Division of groups for estimation of Microalbumin and other Parameters}

$\mathrm{FBS}=$ fasting blood sugar, $\mathrm{PPBS}=$ post prandial blood sugar, $\mathrm{HbA1C}=$ glycosylated hemoglobin, $\mathrm{ADA}=$ American diabetes association

\begin{tabular}{|c|l|c|c|c|}
\hline \multirow{2}{*}{ Sl.No } & \multirow{2}{*}{ Parameters } & Patients & \multirow{2}{*}{ P Values } & Controls \\
\cline { 3 - 4 } & & MEAN \pm SD & & MEAN \pm SD \\
\hline 1 & Microalbumin & $193.5 \pm 73.66$ & $0.0001^{*}$ & $19.06 \pm 3.94$ \\
\hline 2 & FBS & $126.83 \pm 42.48$ & 0.001 & $88.9 \pm 10.78$ \\
\hline 3 & PPBS & $175.06 \pm 64.93$ & 0.001 & $126.3 \pm 27.05$ \\
\hline 4 & Creatine & $2.96 \pm 2.33$ & 0.001 & $0.92 \pm 0.27$ \\
\hline 5 & HbA1C & $7.17 \pm 1.44$ & 0.001 & $5.29 \pm 0.42$ \\
\hline \multicolumn{2}{|c|}{ Table -2: Comparison of Mean and SD in Patients and Controls }
\end{tabular}

FBS = fasting blood sugar, $\mathrm{PPBS}=$ post prandial blood sugar, $\mathrm{HbA1C}=$ glycosylated hemoglobin 
DISCUSSION: The present population based study was carried out to assess the role of association between microalbumin and nephropathy in type- 2 diabetes mellitus, a strong predictor of speed progression towards end stage renal failure.

As duration of diabetes advances in untreated patients it leads to microvascular damage causing various complication one of them being nephropathy. In the present study, the patients were suffering from diabetes mellitus for the past 5 to 10 years. None of them had clinical manifestations of well-developed nephropathy. As shown in the Table -2 increased microalbuminuria of $193.50 \pm 73.66$ $(\mathrm{P}<0.0001)$ is seen when compared with controls subjects $193.06 \pm 3.94$. The above parameters indicate that mild vascular damage starts with the hyperglycemia and hence it is essential that the patients be subjected to periodical assessment of renal functions, the best is to test for albumin in urine. The level of glycemic control seems to be strongest factor influencing transition from normoalbuminuria to microalbuminuria.

Mild increase in fasting blood glucose has been found in serum of diabetic patients $126.83+$ $42.48(\mathrm{P}<0.001)$ when compared with control $88.9 \pm 10.78$.

High fasting blood sugar of long duration can cause microvascular complications like retinal damage leading to vision loss (retinopathy), sensory nerve damage (neuropathy) and when these elevated levels of blood glucose are chronic there is a potential that irreversible kidney damage can occur (nephropathy).

An increased post prandial blood glucose level has been observed in serum of diabetic patients $175 \pm 64.93$ and is not statistically significant when compared to control $126.23 \pm 27.05$ in the study.

Post prandial hyperglycemia has a greater effect on glycosylated hemoglobin $\left(\mathrm{HbA}_{1 \mathrm{c}}\right)$ than fasting glucose levels. Poor PPG control has been associated with development of surrogate markers for cardiovascular disease and has recently been proposed as a risk factor for cardiovascular disease. A number of studies have demonstrated an association between poor PPG control and the development of cardiovascular disease. The International Diabetes Federation (IDF) Guideline for Management of Post meal Glucose provides a good review of these studies. ${ }^{16}$ In particular, the Diabetes Epidemiology Collaborative Analysis of Diagnostic Criteria in Europe (DECODE) study is key study that demonstrated that Post prandial glucose levels better predicted excess mortality due to all causes and cardiovascular events than Fasting plasma glucose levels. ${ }^{17}$

In the study serum creatinine values were not classically elevated to the extent to diagnose renal failure. Though they are elevated in most of the cases $2.96 \pm 2.33(\mathrm{P}<0.001)$ as compared to control $0.92 \pm 0.27$ to the upper part of normal range, they are not significant markers for renal function at sub clinical stage.

Measuring serum creatinine is a simple test, and it is the most commonly used indicator of renal function. ${ }^{18}$

A rise in blood creatinine level is observed only with marked damage to functioning nephrons. Therefore, this test is unsuitable for detecting early-stage kidney damage. A better estimation of kidney function is given by calculating the estimated glomerular filtration rate (eGFR).

Glycosylated hemoglobin values of the patients in the study varied between with mean value of $7.17 \pm 14.4(\mathrm{P}<0.001)$ when compared with controls $5.29 \pm 0.42$ and percentage increase is not significantly seen. The HbA1c level is proportional to average blood glucose concentration over the previous four weeks to three months. 
Poor blood glucose control and persistent elevated glycosylated hemoglobin $\left(\mathrm{HbA}_{1 \mathrm{c}}\right)$ increase the risk of long-term vascular complications of diabetes such as coronary disease, heart attack, stroke, heart failure, kidney failure, blindness, erectile dysfunction, neuropathy (loss of sensation, especially in the feet), gangrene, and also increases the risk of short-term complications of surgery such as poor wound healing.

CONCLUSION: Our study has found prevalence of microalbuminuria in all the patients of type-2 diabetes mellitus indicating subclinical damage of microvasculature, which is the predictor of late development of diabetic nephropathy. Results of our study confirm and extend the previous observation in small selected group of patients with type- 2 diabetes mellitus. But the presence of microalbuminuria alerts the physician to prevent further renal damage by timely administration of ACE inhibitors and correction of risk factors. Urinary excretion of albumin should be monitored routinely in patients with diabetes mellitus.

\section{REFERENCES:}

1. Wild S, Roglic G, Green A, Sicree R, King H. Global prevalence of diabetes: estimates for the year 2000 and projections for 2030. Diabetes Care. 2004; 27:1047-53.

2. Unnikrishnan RI, Rema M, Pradeepa R, Deepa M, Shanthirani CS, Deepa. Prevalence and risk factors of diabetic nephropathy in an urban South Indian population: the Chennai Urban Rural Epidemiology Study (CURES 45) Diabetes Care. 2007; 30:2019-24.

3. Prevalence of small vessel and large vessel disease in diabetic patients from 14 centers. The World Health Organization Multinational Study of Vascular Disease in Diabetics. Diabetes Drafting Group. Diabetologia. 1985;28(Suppl):615-40

4. Sobngwi E, Mbanya JC, Moukouri EN, Ngu KB. Microalbuminuria and retinopathy in a diabetic population of Cameroon. Diabetes Res Clin Pract. 1999; 44:191-96.

5. Sacks DB, Arnold M, Bakris GL. Guidelines and recommendations for laboratory analysis in the diagnosis and management of diabetes mellitus. Diabetes Care 2011; 34-61.

6. Gross JL, Azevedo MJ, Silveiro SP. Diabetic nephropathy: diagnosis, prevention and treatment. Diabetes care 2005; 28:164.

7. Ruggenti P, Remuzzi G. Nephropathy of type-2 diabetes mellitus. J Am Soc Nephrol 1998; 9: 2157.

8. Ismail N, Becker B, Strzelczyk P, Ritz E. Renal disease and hypertension in non -insulin dependent diabetes mellitus. Kidney Int 1999; 55: 1.

9. Mogensen CE. Prediction of clinical diabetic nephropathy in IDDM patients. Alternatives to microalbuminuria? Diabetes 1990; 39: 761.

10. Demaine A, Hibberd M, Millward A. Insertion/deletion polymorphism in the Angiotensin - I converting enzyme gene Diabetologia 1995; 38:1495-96.

11. Le Floch JP, Marre M, Rodier M, Passa P. Interest of Clinitek Microalbumin in screening for microalbuminuria: results of a multicentre study in 302 diabetic patients. Diabetes Metab.2001; 27:36-39.

12. Gansevoort RT, Verhave JC, Hillege HL, Burgerhof JG, Bakker SJ, de Zeeuw D. The PREVEND Study Group. The validity of screening based on spot morning urine samples to detect subjects with microalbuminuria in the general population. Kidney Int Suppl.2005; 94:28-35. 
13. Sarafidis PA, Riehle J, Bogojevic Z, Basta E, Chugh A, Bakris GL. A comparative evaluation of various methods for microalbuminuria screening. Am J Nephrol. 2008; 28:324-29.

14. Estimation of creatinine by the method of Jaffe's kinetic method (text book of clinical chemistry) 3rd ed; Tietz.p.1271 - 80.

15. Goldstein DE, Little RR, Lorenz RA, Malone JI, Nathan DM, Peterson CM. American Diabetes Association. Tests of glycemia in diabetes. Diabetes Care. 2003; 26(Suppl 1):106-08.

16. Schrot RJ. Targeting plasma glucose: Pre-prandial versus postprandial. Clinical Diabetes. 2004; 22(4):169-72.

17. Glucose tolerance and cardiovascular mortality: Comparison of fasting and 2- hour diagnostic criteria. Arch Intern Med. 2001; 161(3):397-05.

18. Taylor E. Howard. Clinical Chemistry, 4thed. New York: John Wiley and Sons; 1989.p. 58-62.

\section{AUTHORS:}

1. Bharathi R.

2. Radhakishan N.

3. Naveen Kumar T.

4. Neetha K.

5. Srilatha B.

\section{PARTICULARS OF CONTRIBUTORS:}

1. Tutor, Department of Biochemistry, Peoples Education Society Institute of Medical Sciences and Research (PESIMSR), Kuppam.

2. Tutor, Department of Medical Biochemistry, Apollo Institute of Medical Sciences and Research, Jubilee Hills, Hyderabad, Andhra Pradesh.

3. Associate Professor, Department of Pharmacology, Apollo Institute of Medical Sciences and Research, Jubilee Hills, Hyderabad, Andhra Pradesh.
4. Assistant Professor, Department of Biochemistry, Apollo Institute of Medical Sciences and Research, Jubilee Hills, Hyderabad, Andhra Pradesh.

5. Tutor, Department of Biochemistry, Apollo Institute of Medical Sciences and Research, Jubilee Hills, Hyderabad, Andhra Pradesh.

\section{NAME ADDRESS EMAIL ID OF THE CORRESPONDING AUTHOR:}

Radhakishan N, Tutor, Dept. of Medical Biochemistry, Apollo Institute of Medical Sciences and Research, Jubilee Hills, Hyderabad, Andhra Pradesh.

Email - kishan.nr@gmail.com

Date of Submission: 17/12/2013.

Date of Peer Review: 18/12/2013.

Date of Acceptance: 03/01/2014.

Date of Publishing: 16/01/2014. 